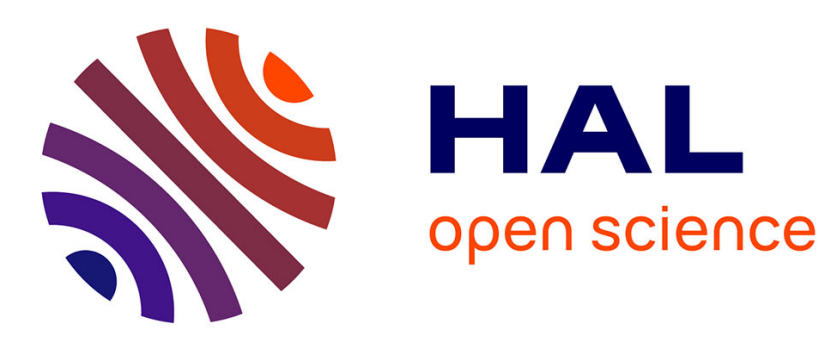

\title{
Paysage urbain : prémisses d'un renouvellement dans la géographie française, 1960-1980
}

\author{
Xavier Michel
}

\section{To cite this version:}

Xavier Michel. Paysage urbain: prémisses d'un renouvellement dans la géographie française, 19601980. Strates: Matériaux pour la recherche en sciences sociales, 2008, 13, pp.67-86. halshs-00840464

\section{HAL Id: halshs-00840464 \\ https://shs.hal.science/halshs-00840464}

Submitted on 2 Jul 2013

HAL is a multi-disciplinary open access archive for the deposit and dissemination of scientific research documents, whether they are published or not. The documents may come from teaching and research institutions in France or abroad, or from public or private research centers.
L'archive ouverte pluridisciplinaire HAL, est destinée au dépôt et à la diffusion de documents scientifiques de niveau recherche, publiés ou non, émanant des établissements d'enseignement et de recherche français ou étrangers, des laboratoires publics ou privés. 
Xavier MICHEL, « Paysage urbain : prémisses d'un renouvellement dans la géographie française, 1960-1980 », Strates [En ligne], 13 | 2007, mis en ligne le 12 novembre 2008, Consulté le 06 juin 2013. URL : http://strates.revues.org/5403

\title{
Paysage urbain : prémisses d'un renouvellement dans la géographie française, 1960-1980
}

\author{
Xavier MICHEL
}

Introduction

Les décennies 1960 et 1970 sont marquées par une évolution de la discipline due à l'engouement pour de nouvelles méthodes, fondées sur la statistique et l'analyse quantitative, puis sur une approche pluridisciplinaire dans les sciences humaines pour traiter de l'espace perçu. Dans le rapport de la géographie à la société, cette vingtaine d'années est aussi une période très importante de mutation de la France. La croissance économique et démographique induit un changement de l'aspect du pays. Les villes existantes s'étendent et se transforment, et d'autres villes sont créées. Beaucoup a été écrit sur les aménagements urbains de cette époque, les politiques, les jeux d'acteurs. Il importe ici de faire le point sur ce qui s'est dit au sujet du paysage urbain. Ce n'est pas une notion nouvelle. Le paysage est classiquement utilisé en géographie, y compris pour qualifier les aspects des villes. Mais jusqu'alors il s'agissait de décrire les centres villes «traditionnels ». L'urbanisation, déjà en cours depuis l'après-guerre, impose aux géographes de tenir compte des effets qu'elle produit sur le paysage. Peu à peu, ils ne vont plus pouvoir penser et décrire le paysage, urbain, comme avant. Une partie au moins des enjeux alors en cours et à venir est intégré par la communauté géographique, et ce dès le début de la période : "...des situations nouvelles, différentes, qui se répercutent sur le paysage urbain comme sur la vie urbaine, ont été créées, qui sont autant de faits géographiques originaux $»^{1}$.

Avant de s'attacher au développement du paysage urbain, deux idées clés apparaissent et permettent de cadrer la réflexion qui fut celle des géographes urbains durant cette période. Tout d'abord, le paysage est représenté comme une notion totalisante. Selon Sylvie Rimbert, cet aspect provient notamment de l'apport de la géographie physique : "Un paysage est un tout, qui se perçoit par bien des sens et dont, si l'on veut le comprendre, on doit débrouiller toutes les relations causales, ainsi que les interactions du complexe vivant qu'il constitue $»^{2}$. Antoine Bailly, autre géographe important dans le champ du paysage urbain, reprend aussi à son compte cette idée, en la puisant cette fois-ci dans la géographie humaine : "Expliquer un paysage d'une manière scientifique, c'est rendre compte des modalités d'interdépendance existant entre ces différents éléments et l'ensemble de leurs caractéristiques »" Trois éléments de la totalité ressortent de ces deux définitions. D'abord, il y a l'aspect d'ensemble : le paysage ne rapporte pas des fragments, mais une physionomie globale de ce qui est perçu. Ensuite, cette perception se réalise par les différents sens, et pas seulement la vue. Enfin, le paysage est une notion qui est faite pour révéler les interdépendances entre ses éléments, ce

\footnotetext{
${ }^{1}$ : GEORGE P., 1961, Précis de géographie urbaine, Paris, PUF, p. 3.

${ }^{2}$ : ROUGERIE G., 1969, Géographie des paysages, Paris, PUF, p. 5, in RIMBERT S., 1973, Approches des paysages, $L^{\prime}$ Espace géographique, $\mathrm{n}^{\circ} 3$, p. 234 . A noter que Sylvie Rimbert ne reprend pas la dernière partie de la phrase : «ainsi que les interactions du complexe vivant qu'il constitue ».

${ }^{3}$ : RACINE J.-B., 1972, La notion de paysage géographique dans la géographie française, Le Géographe canadien, $\mathrm{n}^{\circ}$ 2, pp. 151, in BAILLY A., 1977, La perception de l'espace urbain, Paris, Centre de Recherche d'Urbanisme, p. 21.
} 
que reformule aussi Sylvie Rimbert: "Une étude de paysage nous paraît essentiellement géographique pour au moins deux raisons : en premier lieu parce que la géographie est une synthèse de relations spatiales et que les paysages résultent de la combinaison de facteurs multiples; i ensuite parce que les formes ont d'abord des aspects concrets, sensibles, différents sous chaque latitude... " ${ }^{4}$. Donc ce n'est pas qu'un tableau, mais aussi un moyen d'analyse en géographie. Ces propos des années 1970 expriment une maturation de la réflexion; il importe donc de voir comment est formulée, implicitement ou par des exemples, cette notion dans la décennie précédente. En lien avec cette idée de système, l'intérêt pour le paysage est aussi présent à travers le prisme d'un environnement qui se dégrade à la suite des transformations de l'espace et de l'usage qu'en fait la société : le paysage "vient de traverser sa période démodée qui sentait le peintre du dimanche, pour retrouver, à travers les menaces de pollutions et de nuisances de toutes sortes, une actualité certaine ». Parallèlement à ce rapport à l'environnement et au cadre de vie ${ }^{6}$, on retrouve l'autre référent évoqué ci-dessus, à savoir le cadre pluridisciplinaire dans lequel s'étudie le paysage : «...n'est-on pas arrivé de nouveau à un retour aux paysages, retour suscité par un intérêt convergent de disciplines et par une sorte de mouvement d'opinion? " ${ }^{7}$.

L'objectif est ici de suivre comme fil conducteur les différents aspects et réflexions qui font la spécificité du paysage urbain, en géographie et par rapport aux villes de ces deux décennies. Ainsi, dans un premier temps il importe de discerner ce qui est spécifique à la notion - et aussi à la réalité - de paysage urbain dans les années 1960 et 1970, par rapport aux autres paysages. Après ce moyen de définition en quelque sorte «externe », le but est d'entrer au cœur du paysage urbain, en développant les conditions qui constituent les fondements de cette spécificité, à savoir la relation entre les espaces du quotidien et du hors-quotidien et les mobilités. A la suite de cette étape relevant la causalité de la notion, il s'agit de décrire ce que sont les paysages urbains, et ceci se fait en deux temps : tout d'abord, montrer que le paysage urbain est une composition de différents éléments, et ensuite, relater les différents paysages urbains que les géographes français ont pu identifier et spécifier. Enfin, l'analyse est replacée dans les cadres pluridisciplinaires et disciplinaires. En effet, le paysage urbain a été pensé en géographie en référence à l'histoire des villes, aux démarches architecturales et urbanistiques. En outre, le développement de la géographie «subjective », relative aux perceptions et représentations, s'est fait par des passerelles avec d'autres disciplines de sciences sociales. Vis-à-vis de ces influences, un retour à la question initiale est opéré : quels sont les apports de la géographie?

\section{Le paysage urbain des années 1960 et 1970 et les catégories de paysages}

Une première définition peut être prise chez Sylvie Rimbert : "Le paysage urbain est fait d'un assemblage de formes dont chacune est porteuse de significations et ces dernières sont hiérarchisées. C'est ainsi que ces formes-signes émettent des signaux qui ne sont pas tous perçus au même niveau » 8 . Certes, mais cela ne nous dit rien sur la spécificité du paysage urbain. Les autres paysages, en dehors des villes, sont aussi des assemblages de formes qui ont un sens, possèdent une hiérarchisation, et qui sont différemment perçues. De même, on cherche à montrer la spécificité du paysage urbain par l'idée de personnification : "On dit qu'une cité est triste, gaie, grise, ensoleillée, dynamique ou conservatrice, on lui donne des

\footnotetext{
${ }^{4}$ : RIMBERT S., 1973, Les paysages urbains, Paris, A. Colin, p. 6.

5 : RIMBERT S., 1973, Approches des paysages, L'Espace géographique, n 3, p. 233.

${ }^{6}$ : ROUGERIE G., 1975, Les cadres de vie, Paris, PUF, « le géographe », 264 p.

7 : ROCHEFORT R., 1974, La perception des paysages, L'Espace géographique, n 3, p. 205.

8 : RIMBERT S., 1973, Op. cit., p. 16.
} 
qualificatifs comme à un individu ${ }^{9}$, mais il existe aussi des campagnes dont on dit qu'elles sont grises ou riantes...

\section{Le paysage urbain par rapport aux autres paysages}

L'enjeu est donc l'identification du paysage urbain, et ceci dans un premier temps par rapport aux autres paysages. Tout d'abord, Pierre George énonce brièvement "un certain nombre de critères descriptifs qui le [paysage urbain] différencient du village $»^{10}$. Ces critères sont la maison urbaine, la voirie urbaine, les services, donc un ensemble d'éléments qui personnifient le paysage urbain. Mais au-delà des éléments et des formes, ce sont surtout les relations entre la ville et l'homme qui vont caractériser le paysage urbain : "Qu'il s'agisse du mobilier urbain, des constructions, des voies de circulation, les objets dépassent souvent la taille humaine. La vision du spectateur est donc limitée et les déformations optiques peuvent être importantes suivant l'emplacement où il se trouve ${ }^{11}$. La spécificité ne réside pas, là encore, dans le dépassement de la taille humaine, mais l'autre partie de l'argumentation est plus intéressante. C'est en ville que les relations de l'individu au paysage sont les plus mouvantes, du fait de la densité de composition du paysage urbain et des changements très importants de perception des paysages urbains au cours de déplacements dans cet espace. Les modifications de paysages sont plus immédiates en ville.

Une autre voie a été peut-être davantage suivie, à savoir la description comparative de différents paysages entre eux. Il peut s'agir de définir un paysage en faisant comprendre qu'il ne relève pas du paysage urbain, donc de faire une démonstration par la négative, par exemple au sujet des lotissements de banlieue : «...dans la banlieue parisienne ce nom désigne plus spécialement les quartiers de pavillons entourées de leurs jardins, dépourvus ou presque d'immeubles collectifs, possédant le minimum d'équipements collectifs, de bâtiments publics et de commerces, sans monument en général, sans voie maîtresse, d'une très grande monotonie $»^{12}$. Le géographe se concentre sur un espace donné pour le qualifier.

Un autre mode de différenciation est la comparaison. Ce qui est recherché est alors un effet de complémentarité et de distinction dans la présentation des paysages. La réflexion peut alors se décliner différemment selon les études de cas. Il peut s'agir de la présentation d'espaces contigus dans une démonstration sur l'organisation des espaces d'une agglomération. L'étude intègre alors des échelles différentes. La présentation peut s'attacher à une partie d'une grande agglomération: "situé à la limite d'une agglomération urbaine privée de région, Märkische Vertel a été conçu comme un grand ensemble sans rôle général et uniquement en fonction de ses besoins propres. Il est complété par un centre commercial et administratif (grand magasin, marché, boutiques, poste, etc.) et deux zones industrielles contiguës ${ }^{13}$. Parfois, la description est laissée de côté. Seule la localisation et la situation importent pour différencier deux paysages, à partir du moment où l'on apporte un aspect de celui qui n'est pas urbain, ainsi pour le grand ensemble Auf Den Horst en périphérie de Hanovre : "entre l'autoroute E8 et le Mittelland Kanal, dans le prolongement de la vaste zone industrielle où se succèdent les usines Volkswagen, Continental et Varta ${ }^{14}$. La comparaison se fait aussi pour des espaces plus réduits, mais toujours contigus. Micheline

\footnotetext{
9 : BAILLY A., 1977, La perception de l'espace urbain, Paris, Centre de Recherche d'Urbanisme, p. 24.

${ }^{10}$ : GEORGE P., 1961, Op. cit., p. 72-73.

11 : BAILLY A., 1977, Op. cit., p. 39.

12 : BASTIE J., 1964, La croissance de la banlieue parisienne, Paris, PUF, p. 535.

13 : BRUNET P., P. PINCHEMEL, 1972, Grandes opérations d'urbanisme en République Fédérale d'Allemagne, Annales de Géographie, $\mathrm{n}^{\circ}$, p. 568.

14 : Ibid., p. 571.
} 
Cassou-Mounat en donne deux exemples sur la côte aquitaine. A Biscarosse, il est question de comparer la partie ancienne et la partie récente de la localité : "Le bourg primitif, avec son église à la croisée des routes, ses quelques vieilles demeures bourgeoises et son château, ses petites maisons modestes entourées de jardins et de vignes côtoie étrangement une ZUP dont les immeubles collectifs et le centre commercial évoquent un paysage de grand ensemble urbain ${ }^{15}$. De façon un peu différente, au sujet du doublet Hossegor/Cap-Breton, il s'agit à l'inverse de mettre l'accent sur le fait que le lotissement le plus récent ne constitue pas un paysage urbain : "La croissante récente de Cap-Breton s'est traduite par la prolifération de petits lotissements ; de 1967 à 1971, deux cent lots environ ont été mis en vente. Le contraste n'en est que plus surprenant avec la "ville-parc 》 voisine d'Hossegor dont les " quartiers » révèlent une incontestable unité de conception ${ }^{16}$. De façon plus générale, Philippe Pinchemel fait la comparaison des paysages sur le mode du bilan de ces rapports entre grands ensembles et zones pavillonnaires, en relevant les avantages des premiers : "La construction des grands ensembles s'est faite en réaction contre les abus des zones pavillonnaires [...] ne pas piller les zones agricoles... ${ }^{17}$. La comparaison s'enrichit alors d'une mise en correspondance historique.

La comparaison peut enfin s'effectuer par une relation entre des localités différentes. De ce point de vue, elle a notamment été réalisée au sujet des stations touristiques. L'objectif reste le même : il s'agit de discerner ce qui forme paysage urbain par rapport à ce qui ne l'est pas. Parmi la typologie des stations de sports d'hiver de Rémy Knafou, quatre exemples peuvent être repris afin d'illustrer cette démarche. La Plagne est décrite par ses "bâtiments [...] qui s'étalent en un ruban [...] ils sont presque tous solidaires [...] d'où l'image de "paquebot des neiges $»^{18}$. La situation est semblable à Superdévoluy, qui "s'est développé en un énorme bâtiment unique, qui s'est progressivement allongé au fil des années " ${ }^{19}$. Il insiste ensuite sur Les Ménuires, où l'on trouve une description plus conforme à ce qu'est le paysage urbain : "des bâtiments séparant les parkings des pistes de ski entourent sur trois côtés la grenouillère implantée sur le seul petit replat du versant ${ }^{20}$. On dispose là de différents éléments qui forment une composition constitutive du paysage urbain. Au sujet d'Avoriaz, la description première se veut moins convaincante, à cause notamment du choix du vocabulaire et de la représentation de l'espace : "les bâtiments sont dispersés en plusieurs "villages» [...] Ces "villages» se composent d'immeubles collectifs plus ou moins massifs $»^{21}$. Mais il faut se méfier du vocabulaire employé. Pour des opérations comparables en termes d'aspect et de volume d'urbanisation, on peut trouver l'expression de "cités lacustres $»^{22}$. Donc la description doit primer sur l'expression pour discerner le paysage urbain.

\section{Le paysage urbain par rapport au paysage rural}

De façon plus serrée, les réflexions ont aussi porté sur la mise en évidence du paysage urbain en le comparant, non plus à d'autres paysages bâtis, mais à des paysages ruraux, ou plus généralement au paysage rural. Cela permet à Jean Bastié de préciser la spécificité du paysage

\footnotetext{
15 : CASSOU-MOUNAT M., 1977, La vie humaine sur le littoral des Landes de Gascogne, Paris, Champion, $\mathrm{p}$. 527.

16 : Ibid., p. 574.

17 : PINCHEMEL P., 1980, La France tome 2 Activités, milieux ruraux et urbains, Paris, A. Colin, p. 359.

18 : KNAFOU R., 1978, Les stations de sports d'hiver des Alpes françaises, Paris, Masson, p. 60.

${ }^{19}$ : Ibid., p. 62.

${ }^{20}$ : Ibid., p. 63.

${ }^{21}$ : Ibid., p. 65.

22 : CASSOU-MOUNAT M., 1976, Tourisme et espace littoral : l'aménagement de la côte aquitaine, L'Espace géographique, $\mathrm{n}^{\circ} 2$, p. 141
} 
urbain en prenant le sol pour objet: "Paysage rural et paysage urbain [...], si opposés d'aspect soient-ils, ne sont pas sans rapports. Si dans les paysages urbains il n'est que support, il acquiert en revanche une troisième dimension en fonction des règlements sur la hauteur et la densité des constructions ${ }^{23}$. La différence porte sur la qualité, mais aussi sur la temporalité : "Découpage et affectation du sol urbain sont déterminés en général pour bien plus longtemps, présentent une bien plus grande rigidité et fixité ${ }^{24}$.

A partir de là, il importe pour les géographes d'observer l'intégration ou la coupure entre les paysages, et de ce point de vue, le souci est le même pour les espaces de banlieue comme pour les espaces du tourisme. Dans les deux cas, il y a comparaison de situations entre deux modes de mise en relation. Jean Bastié prend l'exemple du niveau d'intégration de différents paysages bâtis au paysage rural : les grands lotissements, "avec leurs petites maisons basses et leur végétation non ordonnée s'étaient assez bien intégrés au paysage rural. Ils n'écrasaient pas le vieux centre villageois [...] Le grand ensemble au contraire provoque une rupture dans le paysage, par les dimensions de ses bâtiments, leur architecture, leurs matériaux modernes, leurs couleurs, les espaces verts ordonnés. Il est le triomphe de la géométrie et de la ligne droite ${ }^{25}$. La même situation existe dans les stations de la côté aquitaine, si l'on compare, d'une part Mimizan : "les contrastes sont vigoureux entre le cour du vieux village, resté très rural et le nouveau centre communal très moderne ${ }^{26}$, et Hossegor, d'autre part: "témoigne d'un souci plus évident d'adaptation au site forestier. $C$ 'est sans doute la seule station de ce littoral conçue véritablement en fonction du paysage landais $\gg^{27}$. Ainsi, les exemples concrets nous montrent déjà que le paysage urbain est celui qui s'intègre mal ou ne s'intègre pas du tout au paysage rural. L'explication sera précisée plus loin.

Sylvie Rimbert explore une autre piste. Elle prend l'exemple d'un même espace, et examine son évolution dans le temps: Le passage entre deux maisons est également un héritage à caractère rural : c'est le passage vers le jardin potager, vers la basse-cour, avec les brouettes et les pieds boueux. Dans un paysage vraiment urbain, ou bien le passage est remplacé par une porte cochère et couvert par le premier étage, où il disparaît complètement et les murs sont mitoyens $»^{28}$. On se place donc ici dans le cas d'une mutation paysagère. Mais le paysage, c'est surtout la relation entre l'homme et l'aspect du pays qui l'entoure, en tenant compte de ses pratiques et de sa façon de percevoir. La datation des références compte ici pour apprécier l'évolution des repères des géographes. Au début des années 1960, le raisonnement s'opère en lien avec un monde rural encore présent aux marges des villes, et donc l'examen du paysage urbain dans son rapport au paysage rural se fait localement : "Le véritable citadin achète ses carottes et ses poulets, et laisse sa voiture contre le trottoir ${ }^{29}$. Au contraire, au cours des années 1970, la comparaison se fait «à distance », les deux paysages étant représentés comme bien dissociés. C'est alors une différenciation en termes de paysages perçu et vécu qui se développe: "Prenons l'exemple d'une grande ville où les citadins sont continuellement exposés aux affiches publicitaires, à la circulation et à la signalisation routière [...] Vont-ils réagir de la même manière que les ruraux? ${ }^{30}$.

\footnotetext{
23 : BASTIE J., 1965, Le sol, élément primordial du paysage urbain, Annales de Géographie, n 406, p. 709.

24 : Ibid., p. 709.

25 : BASTIE J., 1964, Op. cit., p. 540.

${ }^{26}$ : CASSOU-MOUNAT J., 1977, Op. cit., p. 533.

27 : Ibid., p. 598.

${ }^{28}$ : RIMBERT S., 1967, La banlieue résidentielle de Strasbourg Genèse d'un paysage suburbain, Paris, Les Belles Lettres, p. 117.

${ }^{29}$ : Ibid., p. 117 (on rappelle que la publication est issue d'une thèse de doctorat soutenue en 1963).

30 : BAILLY A., 1977, Op. cit., p. 10.
} 
3. Le paysage urbain des années 1960-1970 par rapport au paysage urbain «traditionnel »

C'est surtout dans la mise en avant du paysage urbain contemporain par rapport au paysage urbain passé que les géographes développent la notion. L'attention est d'abord attirée par le nouvel aspect de la ville: "les nouvelles constructions rompent brutalement avec les traditions architecturales du pays » ${ }^{31}$. D'une part, "l'emploi des matériaux modernes permet de substituer aux anciens paysages urbains un décor tout à fait nouveau [...] surgissent des bâtiments de ciment et de verre qui rompent résolument avec l'harmonie des lignes d'autrefois ${ }^{32}$. Ces matériaux changent par eux-mêmes une partie du paysage, mais ils sont aussi les facteurs d'une mutation plus complète, qui associe l'élévation des immeubles à une nouvelle répartition des surfaces bâties et non bâties: "l'immeuble qui s'élève plus haut qu'autrefois recule par rapport à la chaussée [...], les cours s'ouvrent, leur fonction dans la vie sociale s'épanouit en se transformant et en recevant de la verdure ${ }^{33}$. Les transformations sont frappantes au moment de la découverte des villes par le géographe : "le trait le plus remarquable du paysage qui se crée, c'est sans doute à Francfort, à Hambourg, à Essen, à Düsseldorf, et dans une certaine mesure à Munich, la prolifération des grands immeubles » ${ }^{34}$

Le deuxième fait essentiel est conjoncturel, mais il pose en même temps le paysage urbain des années 1960 comme particulier, et il implique des conséquences. Pierre George rappelle bien la différence et l'originalité du paysage urbain contemporain : "Le passé urbain représentait une somme d'apports successifs échelonnés sur plusieurs décennies, dans certains cas sur plusieurs siècles [...]. La reconstruction, en particulier parce qu'elle s'effectue sous la pression de l'urgence, est au contraire un fait global qui s'inscrit dans un seul moment de l'évolution ${ }^{35}$. La première conséquence est l'existence - éphémère - d'une ville qui n'est pas achevée et déjà habitée : «le grand ensemble conserve longtemps encore après l'arrivée des premiers habitants, une allure de chantiers, quelque chose d'inachevé, avec ses terres défoncées, ses bulldozers, ses grues géantes, les squelettes de ses bâtiments en cours de construction [...]. Les habitants [...] connaissent la boue durant plusieurs hivers $»^{36}$. Ici encore, la correspondance avec le paysage de la station touristique est frappant: "les belles grues oranges resplendissent sous le soleil d'hiver [...] les touristes font le délicat apprentissage $d u$ cheminement dans la gadoue, sautant [...] sur un rocher [...] miraculeusement épargné par les bulldozers » $\$ 7$.

Ensuite, il existe des effets à long terme qui vont marquer le paysage urbain de ces espaces. Sylvie Rimbert nous place d'emblée dans le fond de la question : "Ces signes, comment se mettent-ils en place? Ils sembleraient devoir résulter de créations collectives; or, c'est rarement le cas. Le plus souvent, une minorité d'architectes, d'urbanistes, d'entrepreneurs-techniciens, d'administrateurs, décident du style, de l'implantation, des règlements dont la majorité devra s'accommoder $»^{38}$, et, même si cette organisation n'est pas nouvelle, elle prend une telle ampleur que le type de paysages urbains, y compris et surtout dans leurs dimensions des relations perceptives des habitants, en sont modifiés. Plusieurs géographes dénoncent alors l'aspect que prend ce nouveau paysage urbain : "la standardisation [...] s'accompagne d'une simplification, d'une uniformisation du paysage

\footnotetext{
31 : BARBAZA Y., 1966, Le paysage humain de la Costa Brava, Paris, A. Colin, p. 685.

32 : GEORGE P., 1961, Op. cit., p. 123.

33 : GEORGE P., 1961, Op. cit., p. 132.

34 : CLAVAL P., 1972, La grande ville allemande, Annales de Géographie, n , p. 540.

35 : GEORGE P., 1960, Problèmes géographiques de la reconstruction et de l'aménagement des villes en Europe occidentale depuis 1945, Annales de Géographie, ${ }^{\circ}$, p. 4.

${ }^{36}$ : BASTIE J., 1964, Op. cit., p. 541.

37 : KNAFOU R., 1978, Op. cit., p. 54.

38 : RIMBERT S., 1973, Op. cit., p. 16.
} 
urbain par rapport à l'état antérieur $»^{39}$. La relation avec ce dernier est effectuée sur le mode d'un bilan négatif : "l'impression de densité est accrue par la monotonie du paysage urbain, formé de constructions de moins de trois étages, édifiées en série ${ }^{40}$.

A l'inverse de la monotonie, il est très intéressant de relever que le ou les immeubles qui se détachent trop de l'ensemble préexistant participent positivement ou négativement au paysage urbain. Pour Yvette Barbaza, l'important est que "l'hôtel ou les appartements introduisent dans le paysage urbain un élément architectural qui respecte ou détruise son harmonie, son équilibre [...]. Trop souvent, les nouveaux édifices, quelle que soit leur destination, s'intègrent mal dans le paysage urbain ${ }^{41}$. Dans cette urbanisation touristique récente et rapide de la Costa Brava, la diversité est excessive, elle rompt l'équilibre. Avec une autre situation, celle de l'agglomération londonienne, elle est en revanche bienvenue : "la quasi-totalité de ces ensembles est constituée d'appartements. Durant la période 19191939, la hauteur optimum fut de cinq à six étages. Les blocs étaient le plus souvent en briques rouges, coiffés de vastes toits et disposés sans effort d'imagination autour de cours cimentées [...]. A partir de 1956, le passage à la dimension verticale s'est généralisé ; les immeublestours de 12 à 20 étages.... La prépondérance donnée à l'expansion en hauteur [...] autorise un aménagement très varié de l'espace urbain [...] tout en conservant de vastes espaces libres qui rompent [...] l'impression de communauté et d'espace fermé ${ }^{42}$.

Ainsi, c'est par un emboîtement d'objets et d'échelles que s'est effectuée, dans une première phase, cette appréhension du paysage urbain chez les géographes français. La référence à ce qui n'est pas urbain ne relève pas que de l'identification, mais aussi de la comparaison, de la mise en relation pour mieux comprendre le paysage urbain, ainsi que ses effets, qu'ils soient positifs ou négatifs. Géographiquement, on s'aperçoit que l'observation n'est pas limitée à la France, et que, surtout, elle intègre les espaces qui viennent de s'urbaniser pour le tourisme.

II. Au cœur du paysage urbain des années 1960-1970 : les fondements de la notion

Jusqu'à présent, le paysage urbain a surtout été examiné dans une démarche de repérage par rapport aux autres paysages. Il s'agit maintenant de comprendre ce qui fait plus profondément sa spécificité. Au cours de la recherche, deux champs d'analyse sont apparus très importants : d'une part, la relation entre les paysages du quotidien, du tourisme et de la visite dans la constitution de la notion, et d'autre part les mobilités intra-urbaines.

1. Des paysages forgés par les mobilités intra-urbaines des populations

Les paysages urbains composent des agglomérations à l'intérieur desquelles les usagers se déplacent. Ce n'est pas une activité qui se superpose au paysage urbain, mais bien un fait qui le constitue, en sachant qu'il n'est pas nouveau : "la vie quotidienne d'une ville comporte un constant mouvement de personnes et de marchandises ${ }^{43}$. A partir de là, la mobilité s'analyse en termes de place du piéton et/ou de l'automobile dans la ville. Ces places se concrétisent par des implantations différenciées au sol, en hauteur, voire en souterrain. Compte tenu de la densité des occupations sur une faible surface disponible, cette organisation place le paysage

\footnotetext{
39 : GEORGE P., 1960, Loc. cit., p. 4.

40 : CHALINE C., 1965, Rénovation urbaine et équipement immobilier à Londres, Annales de Géographie, $\mathrm{n}^{\circ}$ 406, p. 715.

${ }^{41}$ : BARBAZA Y., 1966, Op. cit., p. 685.

42 : CHALINE C., 1965, Loc. cit., p. 725-727.

43 : GEORGE P., 1961, Op. cit., p. 126.
} 
urbain comme le résultat du traitement d'un problème : "le problème concret à résoudre consiste à assurer l'accessibilité des immeubles résidentiels à la circulation [...]. Cette organisation est celle de la rue, alignant ses maisons contiguës souvent parfaitement semblables sur des centaines de mètres de long, bordée par un trottoir relativement étroit, qui n'a d'autre raison d'être que de séparer la piste de circulation des piétons et d'accès aux magasins et aux immeubles, de la "chaussée » destinée à la circulation des véhicules ${ }^{44}$. Mais cette question traditionnelle de la répartition des usages du sol est posée en des termes nouveaux dans les années 1960, avec l'essor accru de l'automobile. La bipartition traditionnelle entre la chaussée et le trottoir ne convient alors plus, et les deux solutions envisagées ou présentées par Pierre George, sont, soit la construction de garages en grande quantité, soit la mise en place de secteurs piétonniers, avec "l'obligation pour les usagers de renoncer, dans une partie de leurs occupations, à se déplacer avec leurs véhicules en développant les centres de commerce de détail pour piétons, comme le Lijnbaan d'Amsterdam ou le marché de détail du centre de Stuttgart ${ }^{45}$. A partir de là, cette nouvelle organisation induit une dissociation des paysages urbains, entre ceux marqués par la "circulation générale à l'échelle de l'organisme urbain » et ceux marqués par la "circulation locale à l'échelle du quartier ou de l'îlot ${ }^{46}$. Cette séparation intra-urbaine entre deux types d'espace est nouvelle, et elle a des conséquences sur le paysage urbain : "on obtient une certaine détente de la vie dans le quartier d'habitation $\gg{ }^{47}$.

Une étude de cas s'attache à décrire les formes prises par cette nouvelle situation. Elle est conduite par Pierre Brunet et Philippe Pinchemel à la suite d'un voyage en Allemagne, qui les amène à visiter plusieurs villes. Ils apportent deux types de différenciation, l'un entre deux espaces, l'autre au sujet d'un même espace qui a évolué dans le temps. Une de leurs principales préoccupations est l'observation comparée des quartiers périphériques des grandes villes, en lien avec la place qu'y prend l'automobile. Parmi les nombreuses descriptions, on retient l'opposition entre les nouveaux quartiers de Munich, comme celui de Nordweststadt, où «on retrouve la double circulation, celle des automobiles sur des voies périphériques aux quartiers et légèrement en tranchées qui conduisent à des garages souterrains et celle des piétons sinueuse entre les édifices et franchissant les précédentes par des ponts ${ }^{48}$, et d'autre part ceux de Berlin-Ouest (Märkische Vertel) ou de Hanovre (Auf den Horst), dans lesquels l'automobile n'a pas été dissociée du reste de l'espace; ce qui est souligné est alors l'encombrement des rues par les voitures, et en même temps l'absence de voies piétonnes. Le souci n'est pas seulement de présenter ces répartitions entre les espaces des deux modes de transport, mais de décrire le paysage urbain qui en résulte, ainsi à Munich : "les voitures en stationnement sont rares sauf sur de petits parcs qui jouxtent le noyau commercial de quartier. Le réseau piétonnier est très étendu et soigné entre les immeubles de 6 à 14 étages assez écartés les uns des autres ${ }^{49}$, où l'on retrouve ici à la fois le dimensionnement et la composition formant le paysage.

L'autre approche de la relation entre l'automobile et la marche dans le paysage est diachronique. Le centre ville de Munich a connu une sensible évolution de son organisation : «En 1963, les responsables [...] montraient un des premiers centres de régulation de la circulation par la télévision, les zooms des caméras permettaient d'identifier les voitures fautives aux carrefours proches de l'Hôtel de Ville $»^{50}$. La modernité est ici présente à la fois

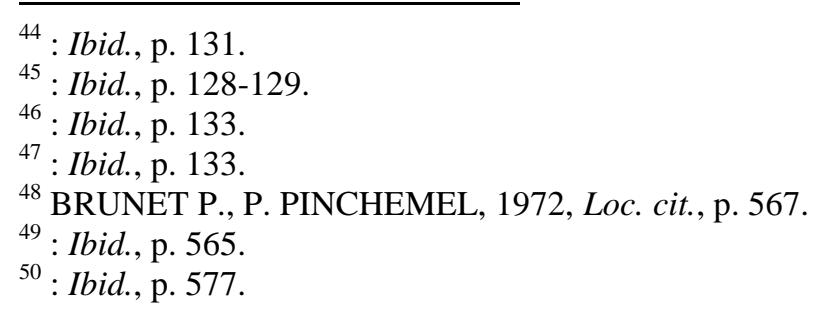


dans le mode de circulation, et dans son mode de contrôle. Huit ans après, le changement est radical : "En 1971, aux mêmes endroits, les rues ont disparu, cédant la place aux artères et places entièrement consacrées à la circulation des piétons $»^{51}$. L'exemple est représentatif d'un mouvement plus général, ainsi au sujet de la City de Norrmalm à Stockholm : "Une certaine réaction vis-à-vis de la voiture amena le nouveau plan de 1967 à modifier dans un sens favorable aux piétons les schémas de circulation; c'est ainsi qu'un réseau cohérent de cheminements piétonniers fut mis sur pied $»^{52}$.

Cette question de la mobilité intra-urbaine dans le paysage est aussi appliquée aux stations touristiques, avec la même sensibilité, par exemple au sujet de Carnon, dans l'Hérault : " son centre [sera] purgé de toute circulation automobile ${ }^{53}$. La description des déplacements permet notamment d'y montrer leurs effets dans le paysage des stations, comme à Avoriaz: "la plupart des immeubles sont accessibles skis aux pieds, mais certains itinéraires exigent d'emprunter des ascenseurs publics, où il arrive, aux heures de pointe des vacances scolaires, que les skieurs fassent la queue! [...] Le boulevard des skieurs est une autre piste de ski intra-urbaine permettant la descente dans l'autre sens ${ }^{54}$. L'analogie avec la ville du quotidien est bien mise en évidence ici, avec le thème de l'accessibilité déjà énoncé par Pierre George, la présentation des encombrements, ainsi que la qualification des espaces à partir de leurs pratiques («le boulevard des skieurs »).

Il sera aussi question de voir que ces mobilités intra-urbaines agissent dans le cadre des espaces perçus et vécus sur le paysage.

2. Des paysages appréhendés par l'association du quotidien, du tourisme et de la visite

A une plus vaste échelle, et avec des relations différentes, le paysage urbain est constitué par l'intégration des espaces du quotidien, du tourisme et de la visite, c'est-à-dire trois espaces-temps qui sont des produits de la civilisation urbaine.

La première posture est de considérer que la ville est l'espace du quotidien, dont il s'agit de s'évader. Après Georges Chabot ${ }^{55}$, c'est Pierre George qui reprend la représentation d'une population voulant s'échapper du cadre urbain, pour aller vers autre chose que la simple campagne environnante: "Réaction significative à l'égard du bâti, le désir d'évasion des citadins d'aujourd'hui. La ville où des générations successives ont vécu sans autre aventure qu'une sortie de temps à autre dans la campagne d'alentour, avec sa signification propre à chaque âge de la vie, est devenue une caserne dont on rêve de "faire le mur » aussi souvent que possible ${ }^{56}$. Ainsi le clivage entre ville et campagne est cassé et recomposé. C'est désormais l'association de la ville du quotidien avec d'autres espaces d'évasion qui importe. A partir de là, ces derniers sont aussi qualifiés de ville : "Sur un territoire de $200 \mathrm{~km}$ de long environ et de $20 \mathrm{~km}$ en profondeur [...] ont été prévues, dès 1963, cinq villes balnéaires nouvelles ${ }^{57}$, ou les villes balnéaires comme Arcachon, Cap-Breton/Hossegor ou Soulac ${ }^{58}$. L'analogie est une démarche alors développée pour mettre en relation des espaces. Ce qui est ici fait penser à ce qui est là-bas, ailleurs. Cela est très imagé : "A la proue du confluent l'usine Chelle qui fabrique des machines à laver les bouteilles aligne à son troisième étage

\footnotetext{
${ }^{51}$ : Ibid., p. 577.

52 : ROUSSET-DESCHAMPS M., 1975, Politique récente d'aménagement urbain à Stockholm, L'Information géographique, $\mathrm{n}^{\circ} 3$, p. 117.

53 : CAZES G., 1972, Réflexions sur l'aménagement touristique du littoral du Languedoc-Roussillon, L'Espace géographique, $\mathrm{n}^{\circ} 3$, p. 196.

54 : KNAFOU R., 1978, Op. cit., p. 66.

55 : CHABOT G., 1957, L'évasion urbaine, La Vie urbaine, avril-juin 1957, 11 p.

56 : GEORGE P., 1965, Géographie et urbanisme, Annales de Géographie, n 406, p. 657.

57 : CAZES G., 1972, Loc. cit. , p. 196.

58 : CASSOU-MOUNAT M., 1976, Loc. cit., p. 133.
} 
une rangée de hublots et introduit une note maritime ${ }^{59}$, ou bien sur un mode plus localisé, avec une comparaison fondée sur la reproduction de modèles, représentée de façon instinctive: "la croissance spontanée du tourisme sur cette côte a eu pour effet la multiplication d'un schéma "instinctif», associant divers éléments empruntés aux villes balnéaires de la Normandie ou de la Côte d'Azur [...]. On en viendrait à confondre Biscarosse et Lacanau, Hourtin-Plage et Saint-Girons ${ }^{60}$, ou bien en révélant une démarche d'imitation: "Arcachon et Soulac sont conçues dans le désir d'imiter les célèbres stations anglaises de la Manche. On a essayé de transposer le plan en damier de Brighton ${ }^{61}$.

Ces villes possèdent les mêmes aspects que l'espace urbain du quotidien, ce qui induit plusieurs réflexions chez les géographes. Tout d'abord, en matière d'aspect de la ville, les mobilités inter-urbaines jouent un rôle fondamental parce qu'elles transforment la présence de la population, avec la "double notion de réseau et de spécialisation des activités [qui] entraîne [...] une sous-occupation urbaine généralisée : la ville touristique est morte en hiver, l'appartement parisien est vide en été ${ }^{62}$. Ce premier point concerne donc la pratique touristique, celle du séjour. Une seconde pratique est mise en évidence : la visite, c'est-à-dire le parcours et la présence en ville pour quelques instants seulement. Elle est énoncée sous l'angle d'une découverte effectuée, non par un touriste, mais par le géographe : "les villes allemandes offrent au visiteur les exemples, réussis le plus souvent, d'une planification physique de leurs espaces actuels et futurs d'urbanisation $»{ }^{63}$. Sylvie Rimbert se place aussi dans cette approche : "A l'angle de la rue de Belfort, le visiteur rencontre avec surprise une famille nombreuse de maisons en rang ${ }^{64}$. Les espaces pratiqués sont plus éloignés du domicile, moins connus, ce qui change le rapport au paysage. Sylvie Rimbert insiste sur l'adaptation que le visiteur doit alors opérer, que celui-ci soit habitant ou touriste: "Ces détails sont cependant moins importants que l'impression de désorientation qui entoure le piéton parcourant ce paysage de grand ensemble. Les lignes directrices $d u$ "plan de masse » sont en effet pratiquement insensibles pour le piéton qui s'égare dans un éparpillement de labyrinthe. Incapable de pouvoir saisir la disposition d'ensemble des blocs, le visiteur qui progresse d'espace vert en espace vert, multiplie les angles de vue au lieu de les ordonner [...]. Il ne se détache aucun bâtiment original sur lequel appuyer la mémoire visuelle, puisque la standardisation en a fait des sosies " ${ }^{65}$. Les statuts et les rôles des individus sont brouillés. Il n'y a plus de paysage du quotidien bien approprié. Les aspects d'ensemble de toutes les villes, du domicile ou de la villégiature, sont comparables. C'est alors sur les détails que le repérage s'effectue : "la vue du touriste est plutôt analytique : c'est le détail qui différencie telle ville de la sienne, qui attire sa curiosité. Le fait que l'un des moteurs du tourisme soit fondé sur l'effet de différence qui contribue à créer le dépaysement est un moyen parmi d'autres de déceler l'originalité d'un paysage urbain ou de saisir la banalité des autres " ${ }^{66}$. Ceci ne signifie pas qu'il n'y a qu'un seul type de paysage urbain, mais pose la similitude entre les éléments récurrents des paysages urbains de la ville du quotidien et de la ville touristique. Et la «vue du touriste » ne se met pas, ou pas seulement, en place parce que l'individu est touriste dans un espace qu'il ne connaît pas, mais elle s'élabore en référence au paysage du quotidien, qui amène spécifiquement l'individu à une perception sur le mode de l'analyse du détail. Comment alors penser la relation entre les paysages urbains du quotidien, du tourisme et de la visite ? Cette relation s'établit en deux temps. Il n'y a pas, d'emblée, une

\footnotetext{
59 : BASTIE J., 1964, Op. cit., p. 528.

${ }^{60}$ : CASSOU-MOUNAT M., 1976, Loc. cit., p. 141.

${ }^{61}$ : CASSOU-MOUNAT M., 1977, Op. cit., p. 598.

62 : RIMBERT S., 1973, Op. cit., p. 56-57.

${ }^{63}$ : BRUNET P., P. PINCHEMEL, 1972, Loc. cit., p. 578.

${ }^{64}$ : RIMBERT S., 1967, Op. cit., p. 114.

${ }^{65}$ : RIMBERT S., 1973, Op. cit., p. 156.

66 : Ibid., p. 45.
} 
différenciation des deux paysages. Au contraire, c'est le processus d'assimilation qui passe en premier, et qui justement, permet ensuite, mais ensuite seulement, la différenciation.

Sylvie Rimbert développe une réflexion sur le paysage urbain comme un paysage de la totalité, au sein duquel les paysages de la ville quotidienne et de la ville touristique forment système : "le dépaysement que recherche le touriste doit [...] provoquer une certaine forme d'inquiétude [...] qui appelle aussitôt des structures sécurisantes, c'est-à-dire habituelles ${ }^{67}$. Elle explicite ainsi la relation entre les espaces sous la forme d'un processus dialectique, qui conduit à une nouvelle étape dans le cheminement de l'individu, le faisant passer de la différenciation à laquelle il était arrivé en repérant des détails dans la ville touristique, à une dé-différenciation qui lui rappelle le quotidien : "On en arrive à transporter son propre paysage urbain quotidien en détruisant l'effet de dépaysement ${ }^{68}$. Serait-on alors revenu au point de départ, à savoir l'assimilation des deux paysages ? Non, car si la matérialité du paysage, autrement dit son cadre, est comparable à l'espace du quotidien, le paysage est aussi constitué de l'animation humaine dans cette matérialité ainsi que des valeurs que l'individu lui confère: "Cette banlieue n'est pas tout à fait semblable à celle de la ville d'origine du touriste ; morphologiquement elle peut même être pire, mais psychologiquement elle peut être parée d'une valeur différente ${ }^{69}$. La dernière étape dans la réflexion est donc celle d'une redifférenciation du paysage urbain touristique par ce qui ne relève pas du matériel dans le paysage. In fine, Sylvie Rimbert en arrive à montrer que le paysage urbain est une totalité, à la fois géographiquement, par l'articulation entre différents espaces-temps humains établis en réseau, et thématiquement, par le fait que ce réseau fonctionne par une intégration, voire une fusion, de la matérialité et des représentations du paysage : "la ville touristique se définit par son opposition à l'environnement courant : on y attend non seulement des formes nouvelles, mais encore des modes de vie, des climats, des ressources, différents de ce que l'on trouve chez soi. Une architecture de ville de vacances ne peut suffire à elle seule à libérer le citadin; il faut la combiner avec de nombreux autres facteurs » ${ }^{70}$.

\section{La description des paysages urbains}

1. Le paysage de la ville est une composition de parties

Différentes parties composent chaque paysage urbain. Antoine Bailly puise chez Kevin Lynch les éléments qui les forment: "La structure du paysage urbain se décompose en cinq éléments : les cheminements, les limites, les nouds, les repères et les quartiers ${ }^{71}$. Mais les géographes, au cours des deux décennies précédentes, ont à maintes reprises exprimé les modes de partition du paysage urbain, dans le but de mettre en évidence sa composition. C'est ce qu'il importe de résumer ici.

Le premier type de description à rapporter est en quelque sorte un « niveau -1 » de description des parties du paysage, car il exprime une partie réelle associée à une partie imaginaire, qui n'existe pas dans le paysage examiné, ainsi à Londres dans Lansdowne Green Estate : "...des immeubles de trois à dix étages...il n'y a pas de réseau intérieur de rues, mais par contre de vastes surfaces dallées. Nul équipement scolaire ou commercial n'a été prévu ${ }^{72}$. Cette absence est aussi relatée dans d'autres descriptions, mais qui, elles, présentent une combinaison de parties bien réelles, par exemple à Hasenbergl, au nord de Munich : «les

\footnotetext{
67 : RIMBERT S., 1973, Op. cit., p. 45.

${ }^{68}$ : Ibid., p. 46.

${ }^{69}$ : Ibid., p. 46.

${ }^{70}$ : Ibid., p. 56.

${ }^{71}$ : BAILLY A., 1977, Op. cit., p. 23.

72 : CHALINE C., 1965, Loc. cit., p. 727.
} 
immeubles à loyer modéré ne dépassent pas huit étages et sont entourés de pelouses et d'arbres. Les équipements correspondent strictement aux besoins primordiaux : trois écoles primaires, une église et un temple avec dépendances communes, un groupe de commerces insuffisants et complétés par des commerces ambulants " ${ }^{73}$. Dans ces deux cas, le géographe renvoie à l'absence d'autres parties, qu'il constate, sinon regrette, lorsqu'il décrit le paysage urbain.

Ensuite, de façon simple, le paysage urbain est décrit comme un ensemble qui rassemble plusieurs parties, en mettant l'accent sur l'association d'un contenant et d'un contenu: "Le centre urbain possède un contenant dans lequel les fonctions urbaines se situent. C'est un assemblage de volumes avec des vides et des pleins qui ont leurs propres caractéristiques. La description toute simple et objective de cet ensemble est importante, il faut connaître l'aspect physique du centre urbain ${ }^{74}$. Il s'ensuit une illustration concrète des éléments possibles. Ce qui importe est l'articulation du volume et de la matière, du physique et de l'humain. A partir de là, le physique ne relève pas que de l'atmosphère, mais aussi de la topographie naturelle préalable à la ville. Cette dernière compose avec elle pour former le paysage urbain, par exemple à propos de la cité satellite de Wallingby à Stockholm : "...et si l'architecture n'est pas d'une grande originalité, les espaces collectifs, aires de jeux, espaces boisés, circuits piétonniers qui utilisent la diversité du paysage fait de collines et de plaines, donnent un paysage urbain très varié ${ }^{75}$. Au-delà d'une apparente simple description, l'auteur met en balance ces différentes composantes pour évaluer la qualité du paysage, ce qui confirme la place du paysage urbain dans le champ des représentations.

Un peu à l'inverse du type initialement présenté - paysage de la présence et de l'absence-, la description du paysage urbain met parfois l'accent sur l'ajout, l'addition d'éléments à un «fond» qui serait plus profondément présent et établirait une première structure dans le paysage : "ces deux artères sont très commerçantes avec leurs cafés fréquentés par les chauffeurs et routiers à la pause de midi [...]. A l'angle des rues Lénine et Molière, un marché moderne aux arcades multicolores en plastique ajoute encore à l'activité commerçante de cet axe ${ }^{76}$. Ici, l'activité de la ville est partie prenante de la «base» paysagère, grâce à une description de paysage de banlieue traditionnelle, et le nouvel équipement est un ajout moderne dans un paysage ayant un certain niveau d'historicité et d'humanité. En revanche, dix ans plus tard, Alain Metton et Michel-Jean Bertrand mettent en évidence une structure centrée fondamentalement sur le bâti neuf, puis élargie aux aménagements entourants, et enfin complétée par la vie des populations : "les logements sont répartis en tours d'une quinzaine d'étages et en petits éléments moins élevés; chacun s'entoure de parkings, pelouses et aires de jeux à la disposition d'une abondante population enfantine ${ }^{77}$. Les nouveaux espaces urbains ne sont pas les mêmes, ils changent le rapport entre l'homme et la ville, et la conséquence sur la description du paysage urbain est nette : la base est devenue le neuf, le béton, qui, avec les aménagements extérieurs, est - ou n'est pasun paysage vécu. La position des différentes parties dans la représentation géographique du paysage urbain est complètement renversée par rapport à la description précédente de la banlieue par Jean Bastié.

Pour approfondir cette périodisation du mode de description du paysage urbain avec ses parties, il s'agit de montrer l'autre renversement de représentation, fondé sur la relation

\footnotetext{
73 : BRUNET P., P. PINCHEMEL, 1972, Loc. cit., p. 570.

${ }^{74}$ : ROUSSET-DESCHAMPS M., 1974, Méthodes d'approche des centres intermédiaires de la banlieue parisienne, Annales de Géographie, ${ }^{\circ} 460$, p. 638.

75 : ROUSSET-DESCHAMPS M., 1975, Loc. cit., p. 119.

76 : BASTIE J., 1964, Op. cit., p. 528.

77 : METTON A., M.-J., BERTRAND, 1974, Les espaces vécus dans une grande agglomération, L'Espace géographique, $\mathrm{n}^{\circ}$ 2, p. 141.
} 
entre les parties et le tout. En effet, au début des années 1960, la représentation du paysage urbain comme une totalité existe, et alors les parties sont énoncées après. Cela est décrit pour la ville traditionnelle: "le corridor commercial canalise quatre axes d'intérêt: $a$ ). l'alignement de façades variées, b). le trottoir à piétons, chiens, voitures d'enfants, c). le caniveau, $d)$. si le trafic de la chaussée est un obstacle aux relations riveraines, du moins entretient-il une distraction continuelle ${ }^{78}$. Mais cette approche du paysage urbain du tout aux parties est aussi appliquée à la description des nouveaux quartiers, comme pour le grand ensemble de la SCIC à Viry-Châtillon : "de loin, il évoque une moderne cité fortifiée, mais lorsqu'on y circule, l'alternance tours, blocs linéaires, petits bâtiments est harmonieux ${ }^{79}$, qui présente un décalage entre un mode de représentation hérité et une nouvelle configuration par rapport à laquelle l'auteur oscille entre surprenance et importunance, pour finalement sembler s'y adapter.

Par la suite, le rapport s'inverse, et la réalité d'espaces urbains nouvellement construits conduit à leur représentation paysagère à partir des parties pour essayer de constituer un tout. Un certain propos de transition est néanmoins formulé au départ, marquant la difficulté relative à sortir du schème précédent, au sujet de Churchill Gardens Estate : "...des immeubles de six à dix étages [...] Des espaces verts abondants, des bandes de verdure cernant chaque immeuble ne dissipent pas l'atmosphère d'une véritable unité de voisinage qui, par ailleurs, possède un équipement complet pour les besoins journaliers " ${ }^{80}$, ou bien exprimant une ville fonctionnelle fondée sur la bonne articulation des parties, à Studley Estate : "Un réseau de circulation pour les automobiles est juxtaposé à un réseau pour les piétons. Une école primaire, des terrains de jeu et une vingtaine de commerces de détail achèvent l'unité fonctionnelle de cet ensemble » ${ }^{81}$. Cette unité fonctionnelle est plus affirmée à partir de l'association des parties dans les nouveaux espaces urbains, comme les stations balnéaires du Languedoc : "intercalation entre les grands ensembles construits de "plaines de sports ", d'équipements de loisirs et d'espaces boisés; structuration des quartiers par la construction de centres commerciaux, de services, d'animation ${ }^{82}$. Mais le géographe n'a pas pour seule fonction de décrire pour décrire. Les partitions des différents paysages urbains, dans une géographie comparée, appellent une évaluation. C'est ce que s'emploient à faire Pierre Brunet et Philippe Pinchemel au cours de leurs excursions urbaines en Allemagne. La composition des parties peut être satisfaisante, à Nordweststadt: "Les immeubles, peu importants [...], sont très variés en hauteur (un à seize étages), et on s'est efforcé de diversifier les espaces intermédiaires en combinant différemment les espaces de jeux propres à chaque groupe de bâtiment, les lieux de repos, les massifs d'arbuste et même les petits jardins qui accompagnent quelques rangées de pavillons. L'école primaire, l'église et des commerces élémentaires se trouvent dans chaque quartier ${ }^{83}$. La qualité du paysage s'obtient par une grande complexité de l'association de ses parties. Dans une situation intermédiaire, d'autres quartiers sont composés de parties qui ne donnent que partiellement satisfaction, comme Märkische Vertel : "quatre lotissements de pavillons très modestes noyés dans les arbres fruitiers de leurs jardinets qui ont été incorporés au plan comme espaces verts, pour les yeux seulement puisque propriétés privées ${ }^{84}$. Enfin, le troisième stade de la description des parties est plus négatif, et il renvoie, non à l'absence, mais à l'insuffisance des parties dans la ville, en relatant le paysage de Limesstadt à Francfort : "Par contre, les espaces non

\footnotetext{
78 : RIMBERT S., 1967, Op. cit., p. 132 (on rappelle la date d'écriture de ces propos : 1963).

79 : BASTIE J., 1964, Op. cit., p. 540.

80 : CHALINE C., 1965, Loc. cit., p. 727.

81 : Ibid., pp. 727-728.

82 : CAZES G., 1972, Loc. cit., p. 198.

${ }^{83}$ : BRUNET P., P. PINCHEMEL, 1972, Loc. cit., p. 567.

84 : Ibid., p. 568.
} 
construits sont peu étendus, le réseau piétonnier assez peu développé et le nombre insuffisant des garages laisse de nombreuses voitures en stationnement de surface ${ }^{85}$. Les nouveaux espaces urbains ont donc amené les géographes à ne plus décrire des ambiances urbaines, dans lesquelles pouvaient apparaître des éléments nouveaux, mais à rendre compte d'aménagements globaux, récents et rapides. Ceux-ci conduisent à des paysages urbains qui s'appréhendent donc d'abord, nécessairement, par leurs parties, et c'est à partir de la plus ou moins bonne réussite de l'articulation entre ces parties qu'une totalité est fondée et que le paysage est qualifié, puis évalué.

Enfin, davantage de sens à cette partition est donné dans les années 1970. En effet, les parties ne servent pas seulement à une composition agglomérée d'un tout, elles sont aussi des éléments qui permettent la perception du paysage urbain. La première approche de cette réflexion s'inscrit dans une représentation statique: "La perception du paysage urbain suppose non seulement la vision d'éléments singuliers (ceux qui, par leur forme, leur fonction ou leur position, se dégagent du tissu urbain) et d'éléments constants (ceux qui, par leur répétition, rendent le tissu urbain homogène), mais aussi [...] l'intégration de l'expérience individuelle ${ }^{86}$. Sylvie Rimbert n'exprime pas ce contraste entre des éléments différenciés qualitativement, mais pour elle l'association des éléments va plus loin que la seule nécessité du repérage: "l'homme construit son repérage sur des oppositions. Les alternances de ruelles étroites et d'échappées lumineuses sont un caractère des paysages urbains [...] Cette psychologie des contraires [...] est absolument nécessaire à une dynamique vitale de mouvements et de déplacements ${ }^{87}$. Le paysage urbain n'est pas seulement le produit des mobilités, il en est aussi une condition. Parler de parties du paysage, c'est également intégrer l'association de la matérialité et de la fluidité, non sur le mode d'une addition, mais d'un fonctionnement systémique.

\section{Les différents paysages du paysage urbain}

Une première série de réflexions générales sur l'organisation de la diversité des paysages urbains dans l'espace des agglomérations peut d'abord être ici restituée. En effet, s'il existe différents paysages urbains, entendus chacun comme la composition de différentes parties, cette diversité est ordonnée, en référence aux agglomérations du quotidien: "en se rapprochant de la ville, les quartiers ont une densité plus forte. La diversité des paysages est plus marquée qu’à la périphérie » ${ }^{88}$, comme aux stations littorales : "...densification de l'habitat vers le centre urbanisé où se concentrent les résidences collectives ${ }^{89}$. La différenciation s'observe plus précisément au sein des villes: "Le centre urbain se différencie des autres types de tissus urbains : zones pavillonnaires, zones industrielles, zones d'ensemble des collectifs " 90 . Outre la densification vers le centre, due à une organisation «libre », une répartition sectorielle des différents paysages s'effectue au sujet des nouveaux quartiers, cette organisation se fondant sur des discontinuités entre les paysages, créées par la planification : "L'établissement d'un plan de zone en 1968 [...] sa principale caractéristique est de fixer les limites des zones d'affaires et des zones d'habitat. La ségrégation est très forte, toute la partie sud de Norrmalm est intégralement occupée par des fonctions tertiaires, bureaux et commerces, c'est le phénomène de city »"${ }^{91}$.

\footnotetext{
85 : Ibid., p. 571.

86 : BAILLY A., 1977, Op. cit., p. 24.

${ }^{87}$ : RIMBERT S., 1973, Approches des paysages, L'Espace géographique, n 3, p. 236.

88 : CLAVAL P., 1972, Loc. cit., p. 542.

${ }^{89}$ : CAZES G., 1972, Loc. cit., p. 198.

90 : ROUSSET-DESCHAMPS M., 1974, Loc. cit., p. 639.

91 : ROUSSET-DESCHAMPS M., 1975, Loc. cit., p. 117.
} 
A partir de ce tableau, les nouveaux espaces urbains donnent lieu à deux principaux niveaux de paysages urbains. Le premier rassemble des paysage traduisant une certaine lisibilité permanente de la ville. Ce sont les paysages de la centralité, mais aussi de la mobilité. Les paysages de la centralité se déclinent, selon l'échelle d'observation, à propos du paysage commercial du centre du quartier : "...la concentration des magasins crée un certain spectacle par l'animation de la clientèle ou la décoration des devantures, et fait apparaître la zone commerçante comme le centre de vie et souvent le symbole du quartier ${ }^{92}$, ou bien, plus largement, au sujet du centre des stations touristiques: "Plus que la plage, le port de plaisance est le principal élément polarisateur des stations nouvelles, la composante majeur de leur paysage, parfois même leur justification essentielle " ${ }^{93}$. Dans les deux cas, le paysage est nouveau ou récent, mais le degré de planification est dans le second cas beaucoup plus abouti. Selon leur échelle et leur mode d'élaboration, les paysages de la centralité prennent donc des ampleurs différentes.

Les paysages aussi bien lisibles et permanents sont ceux de la mobilité. Sylvie Rimbert annonce, en se référant au quartier de La Meinau, l'importance du "paysage des transports : dans les HLM, les automobiles ont droit à presque trois fois plus de terrain que les habitants à qui l'on n'accorde même pas une pièce personnelle: on aboutit à une condensation du banlieusard en hauteur, et à un étalement des voitures en surface " ${ }^{94}$. D'autre part, la mobilité est aussi celle du piéton, produisant un autre paysage urbain : "Un nouveau paysage de la rue apparaît. La grande artère piétonnière est dégagée, parfois très large. Elle forme une promenade entre les magasins et les édifices artistiques $\gg{ }^{95}$. Entre ces deux types bien marqués, et antagonistes, des liaisons typologiques existent, par exemple sur cette description de Stockholm: "Les rues réservées aux piétons sont en général étroites, une dizaine de mètres, créant des espaces intimes animés par la présence de boutiques ou de grands magasins. Les rues affectées aux voitures, plus larges, ont néanmoins des trottoirs suffisamment vastes pour que les piétons ne soient pas incommodés par le trafic [...] La place Sergel est le cour de la ville, important næud de transport en commun, c'est là que les grands magasins se sont installés ${ }^{96}$. On s'aperçoit que la description du paysage par la mobilité ne peut faire abstraction de la centralité urbaine, ce qui, au-delà de la diversité typologique énoncée, renvoie à l'idée de totalité du paysage.

L'autre niveau de description des paysages urbains traduit des paysages moins lisibles, difficiles à percevoir, et/ou qui sont marqués par une évolution rapide, avec des aspects changeants: "Lorsque de fortes densités de population poussent le besoin de partition à l'extrême, on est conduit au paysage urbain du labyrinthe, qui apparaît comme l'une des meilleures solutions à la coexistence de la privatisation et du contact avec la vie publique " ${ }^{97}$. Sylvie Rimbert fait référence à Abraham Moles pour exprimer cette représentation de la ville labyrinthique. Dans un souci qui se poursuit de prendre en compte les présences humaines dans la ville, les paysages urbains les plus appropriés sont alors énoncés : "Ces remarques éclairent l'attrait des paysages urbains de villes historiques méditerranéennes ou nordeuropéennes, et la répulsion générale du piéton pour les grandes lignes droites ou les déserts de vastes dégagements à la Brasilia ${ }^{98}$. C'est le résultat de l'association des mobilités et de la ville qui conduit à ce type de paysage urbain. Le labyrinthe, en apparence le paysage le moins lisible, est en fait celui dans lequel la population se sent le mieux.

\footnotetext{
92 : METTON A., M.-J., BERTRAND, 1974, Loc. cit., p. 144.

93 : CAZES G., 1972, Loc. cit., p. 197.

94 : RIMBERT S., 1967, Op. cit., p. 152.

95 : BRUNET P., P. PINCHEMEL, 1972, Loc. cit., p. 558.

${ }^{96}$ : ROUSSET-DESCHAMPS M., 1975, Loc. cit., p. 118.

${ }_{97}$ : RIMBERT S., 1973, Approches des paysages, L'Espace géographique, n 3, p. 235.

98 : Ibid.
} 
L'autre caractère de manque de lisibilité du paysage provient des changements d'aspect de lieux. Ceux-ci se fondent soit sur une temporalité journalière: "...le fait que le centre d'affaires soit quasi exclusivement occupé par des bureaux et des magasins donne une impression un peu pénible d'abandon lorsque le soir arrive. Ceci explique pourquoi la vieille ville, Gamla Stan, est alors si vivante " 99 , soit sur une temporalité saisonnière : "L'hiver, la neige recouvre tout [...] ce manteau blanc et délicat peut enjoliver un paysage banal [...] la couverture neigeuse peut fort bien rester quatre, cinq, six mois sur un versant nord, mais il n'en est pas de même dans la station où [...] certains éléments se découvrent d'autant plus vite qu'ils sont plus laids à regarder ${ }^{100}$. Au sujet d'espaces urbains très différents, le mode de réflexion du géographe est semblable en ce qui concerne la mise en évidence des effets des temporalités dans la ville sur le paysage urbain : il s'agit d'établir une comparaison entre deux espaces. De façon complémentaire, le mouvement de la nature n'est pas une gêne dans l'affirmation du paysage urbain, bien au contraire : "Dans la deuxième moitié de la saison, lorsque les jours s'allongent et que le soleil devient plus agressif, la station apparaît sous son vrai visage; le village des neiges a disparu, l'embryon de ville a resurgi ${ }^{101}$.

Enfin, le dernier type de paysage urbain est celui qui s'élabore dans la dernière partie de notre période. Il est présenté comme une meilleure solution compte tenu des effets négatifs de ceux qui ont été fondés depuis la fin des années 1950 : "On l'accuse (la charte d'Athènes) de ségréger les différents quartiers, de séparer l'habitat des activités [...] à Cergy-Pontoise comme à Evry, le cour de ville est quasiment réservé aux grands équipements. Les réalisations les plus récentes sont au contraire caractérisées par la polyvalence des fonctions et l'intégration systématique de l'habitat au milieu des activités et des équipements collectifs ${ }^{102}$. Ce paysage urbain nouveau redonne en même temps plus de pertinence à sa composition en différentes parties.

\section{Conclusion}

La géographie française a ainsi pris la mesure des nouveaux paysages urbains en développement dans l'Europe occidentale des années 1960-70. Ce premier point montre que l'appréhension de l'objet et la réflexion sur le sujet se sont notamment fondées sur des exemples étrangers. Pour cela, les difficultés initiales à cerner les mutations et ses enjeux ont peu à peu été comblées par la capacité à mobiliser la réflexion disciplinaire afin de mettre en relation les composantes diverses qui, finalement, ont toujours contribué à faire la géographie, à savoir les différents types d'espaces, leurs aspects et leurs fonctions, leurs matérialités et humanités, et ce dans le présent sans oublier le passé ou, en tout cas, le mode d'antériorité. Néanmoins, au-delà de la spécificité de paysages urbains «neufs », il a aussi fallu, et c'était c'est toujours ?- plus novateur, mettre en avant le rôle des mobilités dans la constitution même de ces paysages. Parmi l'ensemble de l'analyse, c'est en effet une des thématiques principales qui a amené à s'interroger sur le renouvellement même de la notion de paysage urbain.

\footnotetext{
${ }^{99}$ : ROUSSET-DESCHAMPS M., 1975, Loc. cit., p. 118.

100 : KNAFOU R., 1978, Op. cit., p. 54.

101 : Ibid., p. 55.

102 : STEINBERG J., 1979, L'aménagement des villes nouvelles, Annales de Géographie.
} 


\section{Bibliographie}

BAILLY A., 1974, La perception des paysages urbains Essai méthodologique, L'Espace géographique, $\mathrm{n}^{\circ} 3$, pp. 211-217.

BAILLY A., 1977, La perception de l'espace urbain Les concepts, les méthodes d'étude, leur utilisation dans la recherche géographique, Paris, Centre de Recherche d'Urbanisme, 264 p.

BARBAZA Y., 1966, Le paysage humain de la Costa Brava, Paris, A. Colin, 717 p. + XXVII p. de sources et bibliographie, $80 \mathrm{p}$. d'annexes, 5 cartes.

BARRERE P., M. CASSOU-MOUNAT, 1980, Les villes françaises, Masson, 249 p.

BASTIE J., 1964, La croissance de la banlieue parisienne, Paris, PUF, 624 p.

BASTIE J., 1965, Le sol, élément primordial du paysage urbain, Annales de Géographie, $\mathrm{n}^{\circ}$ 406, pp. 708-713.

BASTIE J., B. DEZERT, 1980, Géographie urbaine, A. Colin, 359 p.

BERTRAND M.-J., 1974, Les espaces humains d'un paysage, L'Espace géographique, nº 2, pp. $147-$ 148.

BERTRAND M.-J., 1978, Pratique de la ville, Paris, Masson, 210 p.

BRUNET P., P. PINCHEMEL, 1972, Grandes opérations d'urbanisme en République fédérale allemande, Annales de Géographie, pp. 555-578.

CAPEL H., 1975, L'image de la ville et le comportement spatial des citadins, L'Espace géographique, $\mathrm{n}^{\circ} 1, \mathrm{pp} .73-80$.

CASSOU-MOUNAT, M., 1976, Tourisme et espace littoral : l'aménagement de la côte aquitaine, L'Espace géographique, $\mathrm{n}^{\circ} 2$, pp. 132-144.

CASSOU-MOUNAT M., 1977, La vie humaine sur le littoral des Landes de Gascogne, Atelier de reproduction des thèses/Champion, 906 p. +158 p. de cartes et croquis.

CAZES G., 1972, Réflexions sur l'aménagement touristique du littoral du Languedoc-Roussillon, L’Espace géographique, $\mathrm{n}^{\circ}$ 3, pp. 193-210.

CHABOT G., 1957, L'évasion urbaine, La vie urbaine, 11 p.

CHABOT G., 1970, Vocabulaire franco-anglo-allemand de géographie urbaine, Faculté des Lettres de l'Université de Strasbourg, Ophrys, 69 p.

CHALINE C., 1965, Rénovation urbaine et équipement immobilier à Londres, Annales de Géographie, n 406, pp. 714-732.

CLAVAL P., 1972, La grande ville allemande, Annales de Géographie, pp. 538-554.

GEORGE P., 1960, Problèmes géographiques de la reconstruction et de l'aménagement des villes en Europe occidentale depuis 1945, Annales de Géographie, n 371, pp. 2-14.

GEORGE P., 1961, Précis de géographie urbaine, Pais, PUF, 279 p. 
GEORGE P., 1965, Géographie et urbanisme, Annales de Géographie, n 406, pp. 641-659.

KNAFOU R., 1978, Les stations intégrées de sports d'hiver des Alpes françaises, Paris, Masson, 319 p.

METTON A., M.-J. BERTRAND, 1974, Les espaces vécus dans une grande agglomération, L'Espace géographique, $\mathrm{n}^{\circ} 2$, pp. 137-146.

PIVETEAU J-L., 1973, L'observation directe du paysage et sa place dans la problématique de la géographie urbaine, L’Espace géographique, n 3, pp. 243-246.

RIMBERT S., 1967, La banlieue résidentielle du sud de Strasbourg Genèse d'un paysage suburbain, Publications de la Faculté des Lettres de Strasbourg/Les Belles Lettres, 239 p.

RIMBERT S., 1973a, Les paysages urbains, Paris, A. Colin, 240 p.

RIMBERT S., 1973b, Approches des paysages, L'Espace géographique, n 3, pp. 233-241.

ROCHEFORT R., 1973, La perception des paysages, L'Espace géographique, n 3, pp. 205-209.

ROUSSET-DESCHAMPS M., 1974, Méthodes d'approche des centres intermédiaires de la banlieue parisienne, Annales de Géographie, n ${ }^{\circ} 460$, pp. 625-643.

ROUSSET-DESCHAMPS M., 1975, Politique récente d'aménagement urbain à Stockholm, L'Information géographique, $\mathrm{n}^{\circ} 3$, pp. 109-123.

STEINBERG J., 1980, L'aménagement des villes nouvelles, Annales de Géographie, n 494, pp. 478486. 